\title{
Diet, microbes, and host genetics: the perfect storm in inflammatory bowel diseases
}

\author{
Vanessa Leone $\cdot$ Eugene B. Chang $\cdot$ \\ Suzanne Devkota
}

Received: 18 December 2012/ Accepted: 28 January 2013/Published online: 12 March 2013

(C) Springer Japan 2013

\begin{abstract}
The incidence of inflammatory bowel diseases (IBD), as well as other inflammatory conditions, has dramatically increased over the past half century. While many studies have shown that IBD exhibits a genetic component via genome-wide association studies, genetic drift alone cannot account for this increase, and other factors, such as those found in the environment must play a role, suggesting a "multiple hit" phenomenon that precipitates disease. One major environmental factor, dietary intake, has shifted to a high fat, high carbohydrate Western-type diet in developing nations, nearly in direct correlation with the increasing incidence of IBD. Recent evidence suggests that specific changes in dietary intake have led to a shift in the composite human gut microbiota, resulting in the emergence of pathobionts that can thrive under specific conditions. In the genetically susceptible host, the emerging pathobionts can lead to increasing incidence and severity of IBD and other inflammatory disorders. Since the gut microbiota is plastic and responds to dietary modulations, the use of probiotics, prebiotics, and/or dietary alterations are all intriguing complementary therapeutic approaches to alleviate IBD symptoms. However, the interactions are complex and it is unlikely that a one-size-fits all approach can be utilized across all populations affected by IBD. Exploration into and thoroughly understanding the interactions between host
\end{abstract}

\section{Leone · E. B. Chang}

Department of Medicine, Gastroenterology,

Hepatology and Nutrition, The University of Chicago,

900 E. 57th St., Chicago, IL 60637, USA

\section{S. Devkota $(\square)$}

Research Division, Department of Medicine, Joslin Diabetes Center, Harvard Medical School,

One Joslin Pl., Boston, MA 02215, USA

e-mail: Suzanne.Devkota@joslin.harvard.edu and microbes, primarily in the genetically susceptible host, will help define strategies that can be tailored to an individual as we move towards an era of personalized medicine to treat IBD.

Keywords Inflammatory bowel diseases - Pathobiont . Western diet · Gut microbiota

\section{Introduction}

The prevalence of "New age" Western disorders has increased dramatically worldwide over the past half-century, including obesity, diabetes, colorectal cancer, and inflammatory bowel diseases (IBD) [1-4]. The Centers for Disease Control and Prevention reports that, in the United States alone, 1/3 of adults are obese while 1.4 million adults suffer from IBD $[4,5]$. The battle over why these diseases are on the rise continues to be a topic of debate, but in the advent of new technological advances to probe these questions, recent studies are emphasizing the complexity of these disorders while also bringing to light new avenues to treat them from both a prophylactic and therapeutic perspective.

Inflammatory bowel diseases, both Crohn's Disease (CD) and Ulcerative Colitis (UC) are complex immune disorders that clearly exhibit a genetic basis [6, 7]. Genome-wide association studies (GWAS) have shown that many genes correlate with the development of $\mathrm{CD}$ and $\mathrm{UC}$, however, not every individual presenting with genetic abnormalities will develop disease. This prevents GWAS to predict development and also emphasizes that genetics alone cannot account for the propensity of an individual to develop CD or UC. Other factors, such as environmental triggers (i.e., NSAIDs, smoking, and diet) must play a role 
[8]. Indeed, dietary intake in Western populations has dramatically shifted to a high-fat, high carbohydrate diet over the past-half century, nearly in concordance with the development of disease [9]. These dietary alterations have resulted in a shift in the composite gut microbiota, from both a structure (taxonomy) and function (metagenomic) perspective, that have been shown to play a role in complex disorders such as IBD in both human and animal studies [10-16]. Gut bacteria outnumber human cells by nearly 10-fold and contain an estimated 1,000,000+ genes (whereas humans contain only 23,000) [17, 18]. This highlights that while genetic mutations in the human genome can be associated with IBD development, gut bacteria and their functions cannot be ignored as major players in disease. The combination of genetic predisposition coupled with environmental factors that shift the gut microbiota can result in the "perfect storm" leading to IBD development.

\section{Genetics and environment in IBD etiopathogenesis}

Clinical observations of patients presenting with $\mathrm{CD}$ or UC suggest that genetics play a role in development of disease. Prevalence of either CD or UC varies among different populations, while a positive family history is exhibited amongst first-degree relatives in 6-22\% of affected patients [19]. A greater concordance of IBD has been identified among monozygotic rather than dizygotic twins and syndromes resembling IBD co-segregate in families with rare genetic disorders [20]. Lastly, concordance is exhibited in the type and site of disease among families with multiple affected members $[6,7]$. However, genomewide searches for IBD susceptibility loci have provided the most convincing evidence of a genetic link, identifying 47 risk alleles in UC and 71 in CD [21].

One gene for which SNP's have consistently been associated with $\mathrm{CD}$ risk is the nucleotide-binding oligomerization domain containing 2 (NOD2 also called CARD15) gene located on chromosome 16. The gene is expressed as a cytoplasmic protein that recognizes muramyl dipeptide, a component of bacterial cell wall peptidoglycan [22]. Thus, the NOD2 protein is important in the discrimination between normal intestinal flora and pathogenic bacteria. Pattern recognition by NOD2 initiates the signal transduction that leads to translocation of NFKB to the nucleus, transcription of specific genes and activation of appropriate innate and adaptive immune responses [22]. The most common susceptibility allele is 3020insC, which encodes a truncated NOD2 protein lacking the last 33 amino acids, and shows association with $\mathrm{CD}$ risk both by itself and in combination with SNPs in other genes [23, 24]. While heterozygote individuals are also at increased risk, NOD2 homozygous individuals may have a 20 -fold or more increase in susceptibility to $\mathrm{CD}$, with a particular susceptibility for ileal disease [25]. However, fewer than $20 \%$ of patients with CD are homozygous for NOD2 variants. Since the discovery of NOD2, several additional susceptibility loci have been implicated in IBD: IBD5, IL23R and ATG16L1 [26-28].

While GWAS have provided important insight into the pathogenesis of IBD, NOD2 mutations, for example, only account for $25 \%$ of CD cases in Caucasians, and is not implicated in the pathogenesis of UC. Furthermore, these genetic discoveries do not explain the variation in incidence between different populations or the rising incidence of disease in industrialized countries over the past 50 years [4]. These clues suggest environmental, and perhaps even epigenetic factors, are playing an increasing role in the pathogenesis of IBD. Together, this points to a "multiple-hit" hypothesis in which a genetically susceptible individual encounters a single or series of environmental triggers, which tip the scale of a compensated immune response in favor of dysregulated inflammation.

\section{Gut microbiota and IBD}

Among these critical environmental encounters forming a second or third hit is dietary intake. Evolution, acting through natural selection, represents an ongoing interaction between a species' genome and its environment over the course of multiple generations. When the environment remains relatively constant, stabilizing selection tends to maintain genetic traits that represent the optimal average for a population. However, as environmental conditions change, evolutionary discordance arises between a species' genome and its environment. Stabilizing selection is replaced by directional selection, moving the average population genome to a new set point $[29,30]$. Initially, when environmental changes occur in a population, individuals possessing the previous average genome experience evolutionary discordance [31]. In the affected genotype, this discordance manifests itself phenotypically as disease, increased morbidity and mortality, and reduced reproductive success. There is now growing awareness and evidence that the profound environmental changes in diet that began with the introduction of agriculture and animal husbandry 10,000 years ago occurred too recently on an evolutionary time scale for the human genome to adapt $[32,33]$. In conjunction with this discordance between our ancient, genetically determined biology and the nutritional patterns in contemporary Western populations, particularly of the last 50 years, many of the so-called diseases of civilization have emerged [33, 34].

Our human physiology is not the only entity that has been challenged by changes in dietary practices- the trillions of microbes that live within our intestinal bioreactor have also been forced to adapt. In many cases, these 
adaptations have not been favorable, and provide yet an additional "hit" in the multi-hit theory influencing the incidence of IBD in genetically susceptible individuals and populations (Fig. 1).

Our enteric microflora interacts intimately with our intestinal mucosa and thus is both essential for metabolizing nutrients that we have not evolved to digest on our own, as well as for maturation and education of intestinal immune cells [35]. The metabolic mutualism between host and microbe, when chronically disrupted, can result in aberrant immune responses leading to disease [36]. Gut microbes can stimulate immune responses either by functioning as adjuvants or antigens. As adjuvants they activate innate immune responses, including dendritic cells and other APCs, and as antigens they stimulate the clonal expansion of $\mathrm{T}$ cells that selectively recognize the antigen through their $\mathrm{T}$ cell receptor. For example, genetically susceptible hosts, including IBD patients, and genetically engineered mice with spontaneous mutations have aggressive $\mathrm{T}$ cell responses to luminal commensal bacteria [37, 38]. Although $\mathrm{B}$ cell responses to enteric microbial constituents are exaggerated in CD, UC, as well as in experimental intestinal inflammation, antibodies are not necessary to transmit disease in experimental colitis, and B-lymphocytes seem to be regulatory rather than pathogenic [39, 40]. Antibodies to bacteria have, therefore, been primarily useful as part of diagnostic tests and could potentially identify clinically important subsets of IBD patients who have selective responses to therapeutic agents and have predictable natural histories [41].

Studies of antigen specificity in IBD patientsare lacking, yet several mouse models of disease have provided great insight into the interplay between genetics and environmental factors that contribute to the development of IBD. Experiments in animal models have shown that disease can be transferred to immunodeficient hosts (such as $\mathrm{T}$ celldeficient severe, combined immunodeficient (SCID), $\mathrm{Rag}^{-1-}$ mice, as well as nude rats) via $\mathrm{T}$ cells that respond to commensal enteric bacteria. Cong et al. [42] demonstrated that $\mathrm{T}$ cell clones that respond to cecal bacterial lysates transfer colitis to SCID mice, but that nonspecifically activated $\mathrm{T}$ cells do not. Since then, they have also demonstrated that flagellin-specific $\mathrm{T}$ cell clones can transfer colitis to SCID mice [37].

In over 12 different animal models, colitis and immune activation fail to develop in the absence of commensal bacteria. Additional studies have demonstrated both bacterial species and host specificity for the induction of experimental colitis. Studies utilizing the IL-10 deficient mouse (IL-10 $10^{-/}$), regardless of background strain, have shown that development and severity of spontaneous colitis is dependent upon the milieu of the enteric gut microbiota

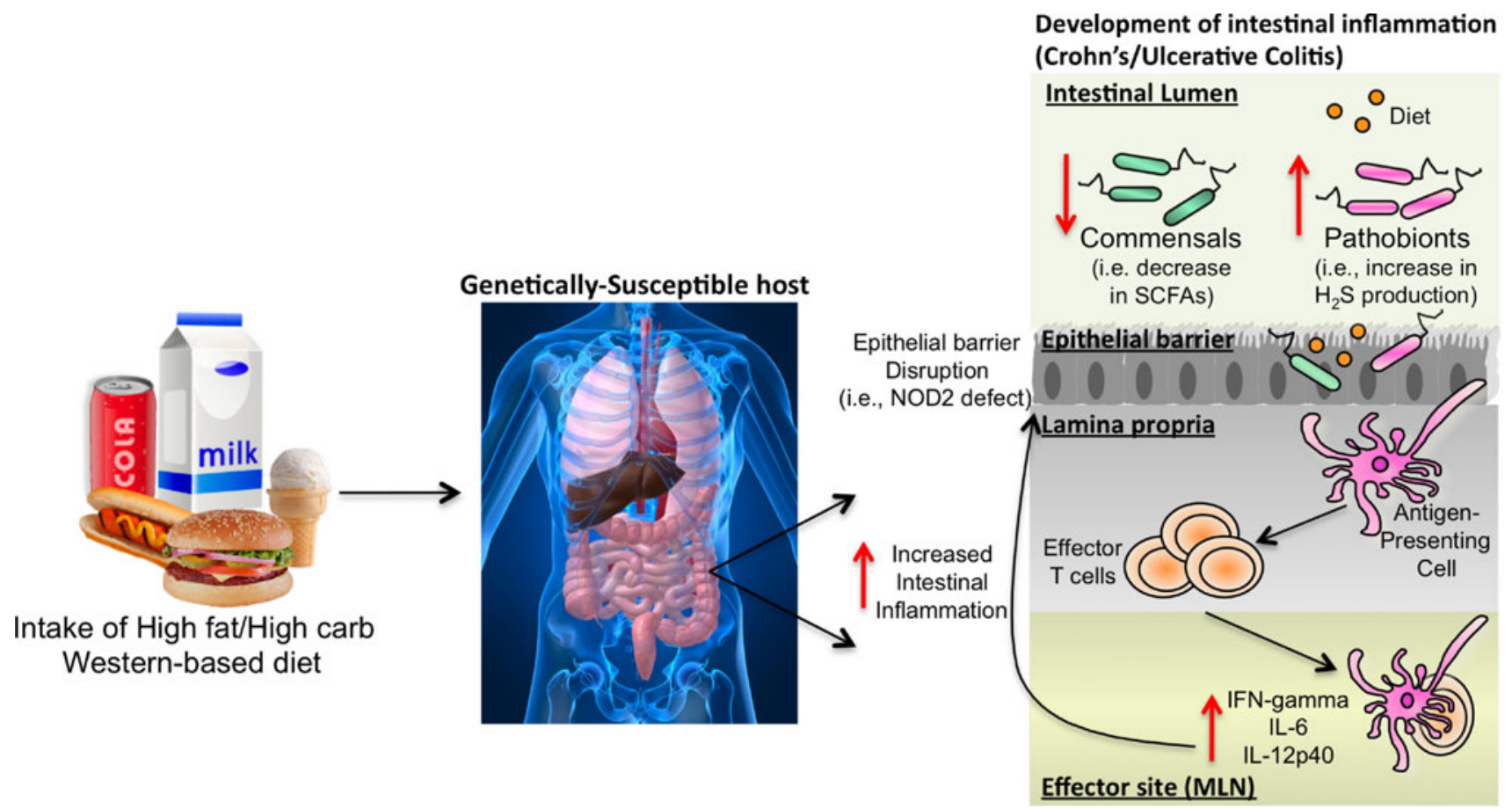

Fig. 1 Intake of Western high fat-high carbohydrate diet promotes expansion of pathobionts in the GI tract, resulting in decreased abundance of commensals. Metabolism of the emerging pathobionts leads to increased host exposure to detrimental bacterial products (i.e., $\mathrm{H}_{2} \mathrm{~S}$ ) and reduced exposure to beneficial products, such as short chain fatty acids (SCFAs). This results in increased immunogenic antigen exposure, prompting inflammatory cytokine production of both antigen-presenting cells (i.e., IL-12p40) and $\mathrm{T}$ cells (i.e., IFNgamma) that increase intestinal inflammation 
$[43,44]$. Penetrance of colitis rises to nearly $100 \%$ in these mice when the enteric microbiota contains Helicobacter hepaticus and is defined by a T-helper 1 (Th1) IFN-gamma response [44]. However, when IL-10 $10^{-1-}$ mice are maintained under germ-free conditions, this mouse does not develop spontaneous colitis [36, 43].

Even in this spontaneous model of colitis, disease can manifest differentially based on the gut bacteria present. Monoassociation of germ-free IL- $10^{-1-}$ mice with the commensal bacteria Bilophila wadsworthia or E. coli induces phenotypically distinct forms of colitis [45]. B. wadsworthia induces an intermediate onset of colitis (3-5 weeks after bacterial colonization) and involves the distal colon, with low-grade colitis accompanied by an exclusively Th1-mediated immune response. Interestingly, the colitis is much more severe when this bacterium blooms in the presence of other bacteria (i.e., specific pathogen free IL-10 ${ }^{-/-}$mice) [36]. In contrast, $E$. coli monoassociation leads to relatively early ( 3 weeks) onset of a mild-to-moderate inflammation that is most severe in the cecum [45]. Preliminary studies show that Klebsiella monoassociation induces moderate pan-colitis and Bifidobacterium animalis monoassociation leads to distal colonic and duodenal inflammation [46, 47]. These results demonstrate that even a traditionally probiotic bacterial species can induce inflammation in a susceptible host, raising concern over the safety of probiotic therapy in some patients.

\section{Gut microbiota: therapeutic potential}

The requirement for host specificity is demonstrated by the variable responses to three unique bacterial species in three different models of colitis: B. wadsworthia and E. coli both increase the incidence of colitis in monoassociated IL$10^{-1-}$ mice, but Bacteroides vulgatus, for example, does not. In contrast, B. vulgatus, but not $E$. coli, induces colonic inflammation in HLA-B27 transgenic rats, and neither causes disease in bone-marrow-transplanted CD3 transgenic mice [45]. Similarly, various probiotic species elicit varying results in the same host, while different hosts respond variably to the same probiotic species [48]. These studies suggest that the disease risk and phenotypes in a single host are highly dependent on the composition of microbiota in the context of different genetic backgrounds. Implied from these observations is the likelihood that personalized therapies for individuals will have to be designed, based on host genetics, biological responses, and gut microbial functional profiles.

In addition, dietary components may become a complementary element of personalized therapies. We know that particular dietary components can alter the composition and virulence of commensals; as stressed earlier, this may provide one potential explanation for the marked increase in the incidence of IBD (and other "New Age" disorders) in Western countries over the last 50 years, and more recently in Eastern countries, as they adopt Western dietary practices [4]. Increased fat consumption is a hallmark of Western dietary practices and has been linked to increased prevalence of IBD. However, many of these studies have only examined the total amount of fat in the diet rather than the fat source. Devkota et al. [36] demonstrated that the type of dietary fats can dramatically reshape the gut microbiota, which can shift the balance of host-microbe interactions in ways that perturb immune homeostasis in genetically-prone individuals and trigger the onset of colitis. The study showed that only the milk fat-based saturated fat diet precipitated disease in genetically susceptible IL-10 $10^{-1-}$ mice by inducing a bloom of the pathobiont B. wadsworthia. However, this finding should not be taken out of this rigorously defined experimental context to suggest that high dietary consumption of milk fat precipitates disease in every individual. The caveat is that environmental and dietary triggers for individuals are highly variable and depend on convergence of numerous factors. A diet high in milk fat in one person may not be the triggering factor for another. The recognition that "New Age" disorders come about by multiple-hit events underscores the importance of personalized medicine in the future where combinatorial factors can be used to assess risk in genetically susceptible individuals.

Similarly, studies have shown that non-absorbed carbohydrates (prebiotics) such as inulin and fructooligosaccharides enhance the growth of beneficial Bifidobacterium and Lactobacillus species providing a substrate for the production of short-chain fatty acids (SCFAs) by these bacterial species. The SCFA's, especially butyrate, are the preferred metabolic substrates of colonocytes, and can stimulate various mucosal barrier functions [46]. Indeed, a study examining twin pairs, with one healthy twin and one with $\mathrm{CD}$ showed that metagenomic and metaproteomic potential was altered in the afflicted twins. Here, reduced diversity was coupled with a reduction in several functional pathways, including carbohydrate metabolism. More specifically, those enzymatic pathways involved in complex carbohydrate metabolism that would result in SCFA production were diminished. In addition, pathways involved in mucin degradation were also decreased in CD patients, indicating that the potential to breakdown oligosaccarhides is diminished during CD [49]. Additionally, a study using methods to predict function via 16s rRNA analyses and validated by shotgun metagenomics on a subset of samples also noted a reduction in pathways involved in SCFAs in CD patients when compared to healthy controls [50]. Morgan et al., correlated their predicted bacterial function analyses to recorded environmental metadata (including 


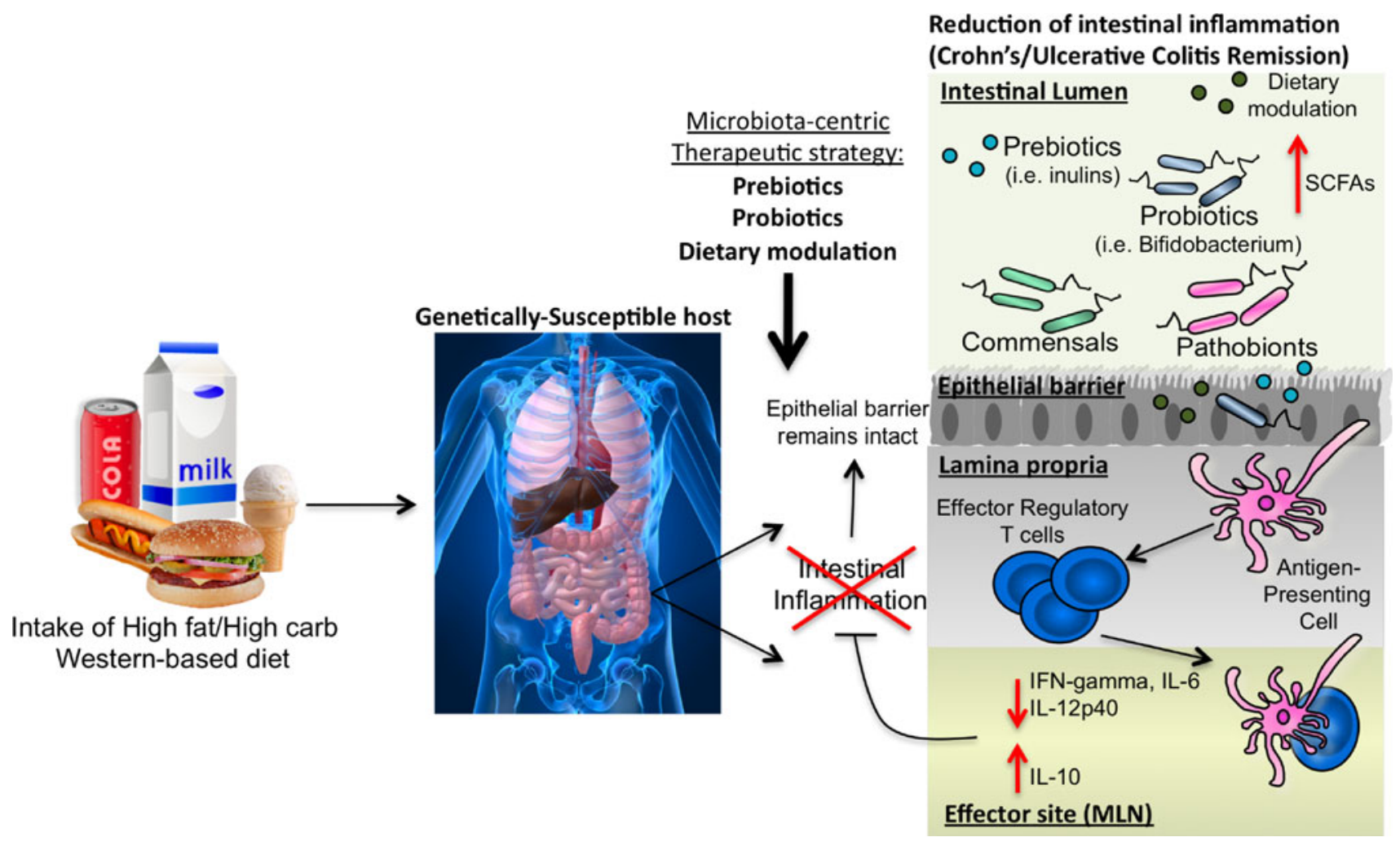

Fig. 2 Microbiota-centric therapeutic strategies, including prebiotics, probiotics, or dietary modulation can promote an increase in short chain fatty acid (SCFA) production even in the context of a Western diet. These therapies may restore commensal microbiota and diminish

smoking and antibiotic/anti-inflammatory drug usage), in patients versus controls, however, neither of these studies tracked dietary intake of these patients and their respective controls. Therefore, interpretation of the effects of CD versus dietary intake or their interaction is difficult. Regardless of the lack of dietary correlations, it would seem that CD patients might benefit from a re-shaping of their gut microbiota to promote metabolic pathways that would increase SCFAs. However, these types of therapies may not benefit all individuals, and clinicians must begin to think about treating IBD at the individual level rather than at a one-size-fits-all level in order to specifically tailor their recommendations.

There is now compelling data to suggest genetically susceptible hosts who are unable to clear an invading pathogen and/or generate tolerogenic immune responses to commensal microbial agents proceed to chronic, relapsing intestinal inflammation. Resistance to $\mathrm{T}$ cell apoptosis, lack of response to downregulatory signals, as well as continuous exposure to luminal antigens and adjuvants help sustain this inflammatory response. It is very likely that environmental factors such as diet may, in turn, profoundly affect mucosal immune responses and enteric bacterial populations. In addition, in an experimental setting utilizing mouse pathobionts. The therapeutic strategies may restore immune balance, promoting anti-inflammatory properties (such as T-regulatory cell function) leading to a decrease of intestinal inflammation in the genetically-susceptible host

models of intestinal inflammation, dietary variation can be carefully controlled, and generally, only one aspect of dietary intake (fat, carbohydrate, or protein) will be modified in a given experiment. Many interactions between dietary components can be missed, especially in the context of the gut microbiota. It is therefore unlikely that one dietary modification will alleviate all symptoms of IBD, and other factors (i.e., lifestyle, medications, etc.) should also be considered. However, once these interactions have been clearly defined and mechanistically described using a variety of human and mouse models of intestinal inflammation, these concepts can be utilized in the clinic as alternative and complementary approaches to prevent/treat IBD, even in the face of Western diets that negatively shift gut bacteria in the genetically susceptible host (Fig. 2).

\section{Conclusions}

The impact of Western diets on gut microbiota and disease outcomes has become increasingly recognized and may account for the observed rising trends in disorders, such as IBD, over the past half century. In the advent of non-culture based techniques to study the gut microbiota, the 
intricate interplay between host genetics and the metagenomic/functional potential of the bacteria can be both explored and exploited. The knowledge gained through further inquiry into the interactions between the host, the environment, and the gut bacteria will help us understand the importance of environmental factors, particularly dietary trends, on human health as they pertain to IBD patients. These concepts can serve as a basis for formulating both prophylactic and therapeutic approaches that are feasible and acceptable to the general population in the era of personalized medicine.

Acknowledgments The authors acknowledge support from NIH/ NIDDK research grants, P30DK42086, UH3DK083993, DK097268, and T32DK07074; NIH/NCAAM research grant F31AT00607.

Conflict of interest The authors declare that they have no conflict of interest.

\section{References}

1. Hotamisligil GS. Inflammation and metabolic disorders. Nature. 2006;444:860-7.

2. Parkin DM, Bray F, Ferlay J, et al. Global cancer statistics. CA Cancer J Clin. 2005;55:74-108.

3. Loftus EV Jr. Clinical epidemiology of inflammatory bowel disease: incidence, prevalence, and environmental influences. Gastroenterology. 2004;126:1504-17.

4. Molodecky NA, Soon IS, Rabi DM, et al. Increasing incidence and prevalence of the inflammatory bowel diseases with time, based on systematic review. Gastroenterology. 2012;142:46-54.

5. Ogden C, Carroll MD, Kit BK, et al. Obesity in the United States, 2009-2010. NCHS Data Brief. 2012;82:1-7.

6. Kirsner JB. The 'idiopathic' inflammatory bowel diseases: their cause and pathogenesis. Arch Dermatol. 1982;118:280-2.

7. Andres PG, Friedman LS. Epidemiology and the natural course of inflammatory bowel disease. Gastroenterol Clin North Am. 1999;28:255-81.

8. Montgomery SM, Ekbom A. Epidemiology of inflammatory bowel disease. Curr Opin Gastroenterol. 2002;18:416-20.

9. de Souza RJ, Swain JF, Appel LJ, et al. Alternatives for the macronutrient intake and chronic disease: a comparison of the OmniHeart diets with popular diets and with dietary recommendations. Am J Clin Nutr. 2008;88:1-11.

10. Turnbaugh P, Bäckhed F, Fulton L, et al. Diet-induced obesity is linked to marked but reversible alterations in the mouse distal gut microbiome. Cell Host Microbe. 2008;3:213-23.

11. Turnbaugh P, Ridaura VK, Faith JJ, et al. The effect of diet on the human gut microbiome: a metagenomic analysis in humanized gnotobiotic mice. Sci Transl Med. 2008;1:6-14.

12. Hildebrandt MA, Hoffman C, Sherrill-Mix SA, et al. High-fat diet determines the composition of the murine gut microbiome independently of obesity. Gastroenterology. 2009;137:1716-24.

13. Walter J, Ley R. The human gut microbiome; ecology and recent evolutionary changes. Annu Rev Microbiol. 2010;65:411-29.

14. Chapman-Kiddell CA, Davies PSW, Gillen L, et al. Role of diet in the development of inflammatory bowel disease. Inflamm Bowel Dis. 2010;16:137-51.

15. Hou JK, Abraham B, El-Serag H. Dietary intake and risk of developing inflammatory bowel disease: a systematic review of the literature. Am J Gastroenterol. 2011;106:563-73.
16. Lepage P, Seksik P, Sutren M, et al. Biodiversity of the mucosaassociated microbiota is stable along the distal digestive tract in healthy individuals and patients with IBD. Inflamm Bowel Dis. 2006;11:473-80.

17. Abdo Z, Shuette UM, Bent SJ, et al. Statistical methods for characterizing diversity of microbial communities by analysis of terminal restriction fragment length polymorphisms of $16 \mathrm{~S}$ rRNA genes. Environ Microbiol. 2006;8:929-38.

18. Cani PD, Delzenne NM. The gut microbiome as therapeutic target. Pharmacol Ther. 2011;130:202-12.

19. Monsen U, Bernell O, Johansson C, et al. Prevalence of inflammatory bowel disease among relatives of patients with Crohn's disease. Scand J Gastroenterol. 1991;26:302-6.

20. Tysk C, Lindberg E, Jarnerot G, et al. Ulcerative colitis and Crohn's disease in an unselected population of monozygotic and dizygotic twins. A study of heritability and the influence of smoking. Gut. 1988;29:990-6.

21. Khor TO, Huang MT, Kwon KH, et al. Genetics and pathogenesis of inflammatory bowel disease. Nature. 2011;474:307-17.

22. Ogura T, Bonen DK, Inohara N, et al. A frameshift mutation in NOD2 associated with susceptibility to Crohn's disease. Nature. 2001;411:603-6.

23. Maeda S, Hsu LC, Liu H, et al. Nod2 mutation in Crohn's disease potentiates NF-kappaB activity and IL-1beta processing. Science. 2005;307:734-8.

24. Gearry R, Roberts RL, Burt MJ, et al. Effect of inflammatory bowel disease classification changes on NOD2 genotype-phenotype associations in a population-based cohort. Inflamm Bowel Dis. 2007;13:1220-7.

25. Lesage S, Zouali H, Cezard JP, et al. CARD15/NOD2 mutational analysis and genotype-phenotype correlation in 612 patients with inflammatory bowel disease. Am J Hum Genet. 2002;70:845-57.

26. Duerr RH, Taylor KD, Brant SR, et al. A genome-wide association study identifies IL23R as an inflammatory bowel disease gene. Science. 2006;314:1461-3.

27. Hampe J, Franke A, Rosenstiel P, et al. A genome-wide association scan of nonsynonymous SNPs identifies a susceptibility variant for Crohn disease in ATG16L1. Nat Genet. 2007;39:207-11.

28. Peltekova VD, Wintle RF, Rubin LA, et al. Functional variants of OCTN cation transporter genes are associated with Crohn's disease. Nat Genet. 2004;36:471-5.

29. Gould SJ. The structure of evolutionary theory. Cambridge: Harvard University Press; 2002.

30. Cordain L, Eaton SB, Sebastian A, et al. Origins and evolution of the Western diet: health implications for the 21 st century. Am J Clin Nutr. 2005;81:341-54.

31. Boaz NT. Evolving health: the origins of illness and how the modern world is making us sick. New York: Wiley; 2002.

32. Eaton SB, Konner MJ. Paleolithic nutrition. A consideration of its nature and current implications. N Engl J Med. 1985;312:283-9.

33. Eaton SB, Konner M, Shostak M. Stone agers in the fast lane: chronic degenerative diseases in evolutionary perspective. Am J Med. 1988;84:739-49.

34. Frassetto L, Morris RC Jr, Sellmeyer DE, et al. Diet, evolution and aging-the pathophysiologic effects of the postagricultural inversion of the potassium-to sodium and base-to-chloride ratios in the human diet. Eur J Nutr. 2001;40:200-13.

35. Backhed F, Ley RE, Sonnenberg JL, et al. Host-bacterial mutualism in the human intestine. Science. 2005;307:1915-20.

36. Devkota SD, Wang Y, Musch MW, et al. Dietary-fat-induced taurocholic acid promotes pathobiont expansion and colitis in Il10 ${ }^{-/-}$mice. Nature. 2012;487:104-8.

37. Lodes MJ, Cong Y, Elson CO, et al. Bacterial flagellin is a dominant antigen in Crohn's disease. J Clin Invest. 2004;113:1296-306. 
38. Hoentjen F, Sartor RB, Ozaki M, et al. STAT3 regulates NF- $\mathrm{B}$ recruitment to the IL-12p40 promoter in dendritic cells. Blood. 2005;105:689-96.

39. MacPherson A, Khoo UY, Forgacs I, et al. Mucosal antibodies in inflammatory bowel disease are directed against intestinal bacteria. Gut. 1996;38:365-75.

40. Mizoguchi E, Mizoguchi A, Chiba C, et al. Anti-neutrophil cytoplasmic antibodies in T-cell receptor alpha-deficient mice with chronic colitis. Gastroenterology. 1997;113:1828-35.

41. Sartor RB. Therapeutic manipulation of the enteric microflora in inflammatory bowel diseases: antibiotics, probiotics and prebiotics. Gastroenterology. 2004;126:1620-33.

42. Cong Y, Weaver CT, Lazenby A, et al. Bacterial-reactive T regulatory cells inhibit pathogenic immune responses to the enteric flora. J Immunol. 2002;169:6112-9.

43. Sellon RK, Tonkonogy S, Schultz M, et al. Resident enteric bacteria are necessary for development of spontaneous colitis and immune system activation in interleukin-10-deficient mice. Infect Immun. 1998;66:5224-31.

44. Kullberg MC, Ward JM, Gorelick PL, et al. Helicobacter hepaticus triggers colitis in specific-pathogen-free interleukin-10 (IL-10)deficient mice through an IL-12- and gamma interferon-dependent mechanism. Infect Immun. 1998;66:5157-66.
45. Kim SC, Tonkonogy SL, Albright CA, et al. Variable phenotypes of enterocolitis in interleukin 10-deficient mice monoassociated with two different commensal bacteria. Gastroenterology. 2005;128:891-906.

46. Sartor RB. Microbial influences in inflammatory bowel disease: role in pathogenesis and clinical implications. In: Sartor RB, Sandborn WJ, editors. Kirsner's inflammatory bowel diseases. Philadelphia: Elsevier; 2004. p. 138-62.

47. Moran JP, Walter J, Tannock GW, et al. Bifidobacterium animalis causes extensive duodenitis and mild colonic inflammation in monoassociated interleukin-10-deficient mice. Inflamm Bowel Dis. 2009;15:1022-31.

48. Dieleman LA, Goerres MS, Arends A, et al. Lactobacillus GG prevents recurrence of colitis in HLA-B27 transgenic rats after antibiotic treatment. Gut. 2003;52:370-6.

49. Erickson AR, Cantarel BL, Lamendella R, et al. Integrated metagenomics/metaproteomics reveals human host-microbiota signatures of Crohn's disease. PLoS ONE. 2012;7:e49138.

50. Morgan XC, Tickle TL, Sokol H, et al. Dysfunction of the intestinal microbiome in inflammatory bowel disease and treatment. Genome Biol. 2012;13:R79. 